longer anæesthesia after the removal of the mask can be obtained with chloroform than with any other anæsthetic, but I maintain that in order to obtain that increase of time the drug must be pushed to the extent of abolishing the cough reflex or perilously near it, and that an anæsthesia which stops short of that point has no advantage whatever over ether narcosis and is infinitely more dangerous.

What, then, are the alternative methods? Infants aged less than 12 months should be given ether on an open mask. Children from one to four years of age do not take $\mathrm{N}_{2} \mathrm{O}$ well, especially if they have large tonsils, but, as a general rule, they take ether without any trouble at all from a Clover's inhaler, with or without the bag, according to the ease with which they go under. For children from four to 12 years of age $\mathrm{N}_{2} \mathrm{O}$ will generally do, but if when pressed it causes much jactitation and rigidity it is better to give a little ether. In adolescents and adults the operation can be done under gas alone in most cases; indeed, a rapid operator will frequently remove in the time allowed by $\mathrm{N}_{2} \mathrm{O}$ anæsthesia tonsils, adenoids, and the posterior ends of the inferior turbinals. What a rapid operator will do under gas only an average operator will do easily under gas and ether.

Gas and oxygen is perhaps slightiy better than gas or gas and air, but for these operations the apparatus is rather inconvenient, and, as a matter of fact, we find that gas or gas and ether meet our demands. The purified chloride of ethyl used as a general anæsthetic is still under trial, but from the results I have had in about 80 cases $I$ doubt if it has any advantage over ether, and it is certainly less trustworthy and more tedious to administer.

What do the critics of these methods say? Their first objection is that the operation cannot be satisfactorily and completely done under this form of anresthesia. Now, this method has been in vogue at the Metropolitan Throat, Nose, and Ear Hospital and at the Central London Throat Hospital for 12 years, during which time 20,000 cases have been so operated upon. Is it seriously suggested that the surgeons attached to these two hospitals have not learnt in 12 years whether or not they can clear a naso-pharynx of tonsils and adenoids in the time allowed by a gas or gas and ether anæsthesia? If at these hospitals these operations were habitually only half done would there not be an ever-increasing back-flow of recurring cases? As a matter of fact, the percentage of recurrences is very small and certainly not greater than that of cases done under chloroform elsewhere. The second objection which is raised is that gas and gas and ether cause more hæmorrhage than chloroform. Even supposing that they do so, unless it can be shown that they cause dangerous hæmorrhage the objection ought not to be taken into account against the risk to life which I have shown is entailed by the employment of chloroform, but, personally, I believe the objection to be purely fanciful. I can only say that amongst the several thousands of these operations which I have seen and assisted at I have never seen a case of alarming or even of serious hæmorrhage. We are told that while chloroform kills on the table, ether kills by its after-effects, and with prolonged administrations this is doubtless sometimes true ; but the objection does not, of course, apply to short inhalations of ether such as are employed for the operations in question.

Gag - The best gag for these cases is certainly Doyen's, and it should be applied before the administration of the anæsthetic.

Position.-For gas or gas and oxygen the sitting position is very much the best. For ether or gas and ether some surgeons prefer the recumbent position, and it is not a matter of much importance. From an anæsthetist's point of view I prefer the sitting position as being more cleanly and as obviating the necessity of using mops or sponges.

The other operations on the list can all be done under gas, gas and oxygen, or gas and ether, and there is never any necessity for the use of chloroform.

In conclusion, I should like to explain that I do not suggest that the methods which $I$ here advocate are confined to the two hospitals named; I only quote them because $\mathbf{I}$ have personal experience of their work and access to their records. Nor do $I$ wish to be misunderstood in my attitude towards chloroform, which in suitable cases I regard as facile princeps of anæsthetics. The points that I wish to emphasise are: (1) chloroform has been the means of destroying more than 50 lives during adenoid operations and cannot, therefore, be regarded as other than a dangerous anresthetic in this particular class of case; (2) at least five of these deaths have occurred in the practice of anæsthetists of the highest possible skill and experience; and (3) thousands of these operations are performed every year under gas and gas and ether without a single death and with hardly an anxious moment. In view of these facts, are we justified in subjecting patients to the absolutely unnecessary risk of chloroform anæsthesia? I think decidedly not.

Welbeck-street, $\mathbf{W}$.

\section{A CASE OF TRAUMATIC RUPTURE OF THE SPLEEN ; REMOVAL FOLLOWED BY EMPYEMA AND RECOVERY.}

BY EDGAR BEAUMONT, L.R.C.P. LOND., M.R.C.S. ENG., SURGEON TO THE NORWOOD COTTAGE HOSPITAL; AND

EDWARD HOUSEMAN, M.B., B.C. CANTAB., LATE HOUSE SURGEON TO ST. GEORGE'S HOSPITAL.

THE patient was a youth, aged 17 years, who was brought into the Norwood Cottage Hospital on the evening of May 20th, 1902. There was a history of his having fallen from a wagon, one wheel of which passed over his body. On examining the youth we found that he was suffering great pain in the left loin. There was a bruise over the ninth and tenth ribs in the posterior axillary line of the size of the palm of a man's hand. His temperature was $97.6^{\circ} \mathrm{F}$, his respirations were 24 , and his pulse was 100 . His breathing was not markedly thoracic and there was an absence of rigidity of the abdominal muscles. There was no evidence of fractured ribs, but there was dislocation of the acromial end of the clavicle and a fracture across the infra-spinous fossa of the scapula with a large hæmatoma under the bone. A few hours after admission the boy became very restless and the distension of the abdomen, with loss of liver dulness, brought forcibly to our minds the fact that there was severe abdominal lesion and decided us to open the cavity without delay. Chloroform and ether were administered and we were then able to feel distinctly a loose body in the left hypochondrium which we took to be, and which ultimately turned out to be, a portion of the spleen. In consequence of the loss of liver dulness we thought it likely that there might be intestinal injury as well, so we decided on a median incision.

We made a free incision in the middle line and on opening the peritoneum found the cavity full of blood-clot. In addition there was a welling up of blood, showing that active hæmorrhage was still going on. There was no free gas in the cavity. On pressing the hand into the cavity the spleen was instantly felt to be in two pieces, and on finding and holding the vessels between the finger and thumb the hæmorrhage was controlled. The spleen was then drawn forward to the surface, the pedicle was clamped, and after separating the tail of the pancreas from the hilum was transfixed, tied, and divided and the spleen removed. The abdominal cavity was then flushed with several gallons of hot sterilised water and the abdomen closed. There was considerable ecchymosis of the intestines.

The patient by this time had become very collapsed, so 15 minims of liquor strychnix were injected hypodermically before he was removed from the table and hot saltwater injections per rectum with five minims of liquor strychniæ hypodermically were continued every three hours during the night. Next morning we found the patient fairly comfortable; the pulse, which had been 160 after the operation, had come down to 120 . He had not vomited, but there was still absence of liver dulness and the patient lay on his back with his knees drawn up. He was unconscious and moaned incessantly. His temperature was $1015^{\circ}$. He remained in much the same condition until the third day after the operation, when his temperature suddenly shot up to $105^{\circ}$. He became violently delirious, restless, and sweated profusely; there was diarrhoea and urine was passed under him, so we decided to dress the wound, which we found apparently healthy. The abdomen was slightly distended but moved freely and did not seem tender. On the sixth day after the operation we found some fluid effused at the base of the left lung, so we aspirated and removed six ounces of decomposing blood with an almost fæcal smell. As there had been no. 


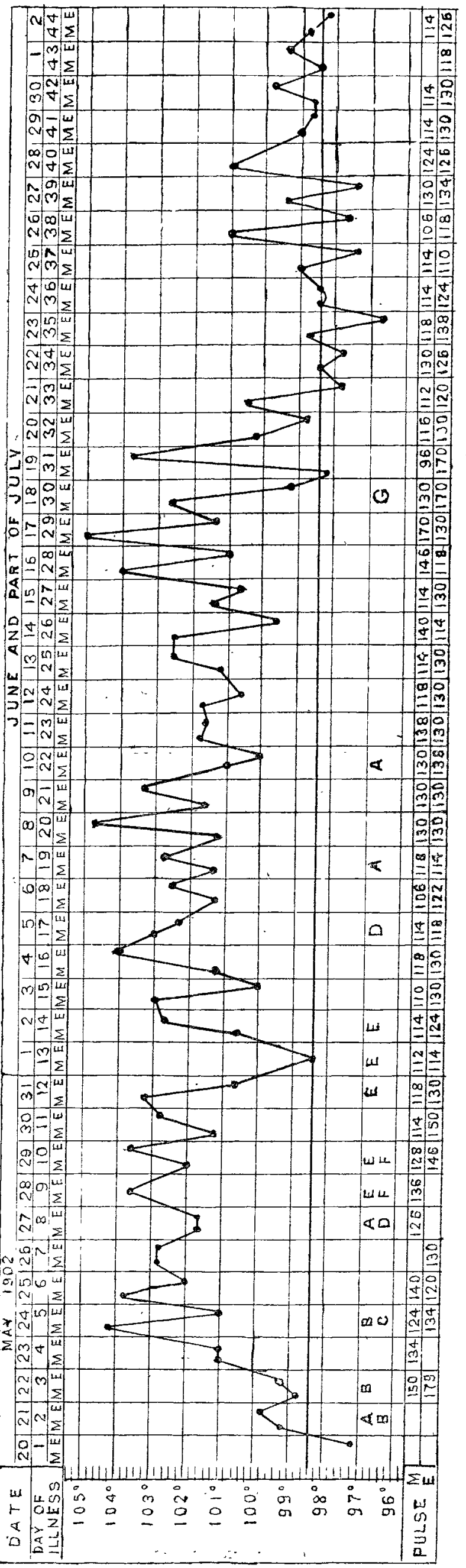

fracture of ribs at the time of the accident we concluded that this had probably come through the diaphragm from the abdominal cavity, and so we decided to explore the subdiaphragmatic region.

With this object we made an incision in the loin from behind forwards, the centre of the incision being over the angle of the eleventh rib. We resected the lower ribs and so were enabled without difficulty to examine the stump of the spleen. Not finding any pus we closed the opening. On this we concluded that septicæmia was the cause of his high temperature and general condition, so we made an examination of the blood and found streptococci in large numbers. We therefore commenced the hypodermic injection of antistreptococcic serum and gave him 90 cubic centimetres with little effect on his temperature, and within four hours he developed a very large pleural effusion on the left side only, the dulness extending up to the clavicle, so we aspirated and withdrew two pints of fluid slightly blood-stained and sweet. We continued the injection for four days, during which time we used 330 cubic centimetres of the serum. On the morning of the fifth day after these injections had been commenced the temperature came down to normal and the patient was quite conscious for the first time for nine days. All the wounds, including the hypodermic punctures, became about this time septic in spite of antiseptic dressings, so we swabbed the worst of them with pure carbolic acid. After this treatment they cleaned up very much. On June 5th, 15 days after the original operation, we found signs in the left chest about the angle of the scapula of a localised empyema, so we aspirated and found pus and subsequently resected considerable portions of the seventh and eighth ribs. There was free discharge of pus for several days, but the temperature remained high, reaching $104^{\circ}$ and $105^{\circ}$ daily. The cavity was washed out with a weak solution of lysol and remained sweet, but as the temperature remained high we decided to try the effect of the frequent application of pure oxygen through a tube passed into the pleural cavity. This treatment was attended by the most satisfactory results. The temperature came down to normal, the discharge ceased, and a general repair commenced in all the wounds. With the exception of the wound leading into the pleural cavity all are now healed; this is rapidly closing and the lung is expanding well and filling the cavity. The patient is in good-general condition, eats and sleeps well, and spends his time in the garden.

Remarks. - The following points are of interest in this case. 1. The absence of collapse and comparative slowness of pulse in so severe an abdominal injury. 2. That since the removal of the spleen the pulse has never come below 112, although the patient is now in comparatively good health. 3. We have noticed that since the removal of the spleen there has been marked general enlargement of lymphatic glands with anæmia. 4. The very slight reaction to exceptionally large doses of anti-streptococcic serum, although to get different strains we obtained it from different sources. 5. The extraordinary general and local improvement which took place at once on the application of oxygen to the pleural cavity.

addendum,--At this date (Sept. 8th) the patient is perfectly recovered and is to all appearances in as good health as before the accident. $\mathrm{He}$ is occupied in light gardening, \&c. His pulse rate still remains, however, at 1rom 112 to 120 .

\section{Clinical 算otes:}

\section{MEDICAL, SURGICAL, OBSTETRICAL, AND THERAPEUTICAL.}

\section{A PEOULIAR CASE OF SCARLATINA} HÆMORRHAGICA.

By J. Duncan Howe, M.R.O.S. Eng.

FORMERLY ASSISTANT MEDICAC OFFICER, MONSALL FEVER HOSPITAL, MANCHESTER,

THE following are notes on a case similar to the one reported by Dr. C. De Boinville in "THE LANCET of August 9th, 1902, p. 359.

The patient, a boy, aged six years, suffering from necrosi, of the right tibia, had been an inmate of the Cheadle 